\title{
Commentary: Neoadjuvant checkpoint inhibitors in resectable non-small cell lung cancer-Ready for prime time?
}

\author{
Boris Sepesi, $\mathrm{MD},{ }^{\mathrm{a}}$ and Tina Cascone, $\mathrm{MD}, \mathrm{PhD}^{\mathrm{b}}$
}

\footnotetext{
From the Departments of ${ }^{\mathrm{a}}$ Thoracic and Cardiovascular Surgery and ${ }^{\mathrm{b}}$ Thoracic and Head and Neck Medical Oncology, The University of Texas MD Anderson Cancer Center, Houston, Tex.

Disclosures: Dr Cascone has received speaker's fees from the Society for Immunotherapy of Cancer and BristolMyers Squibb, receives consultant fees from MedImmune and Bristol-Myers Squibb, and receives research funding to MD Anderson Cancer Center from Boehringer Ingelheim, MedImmune, and Bristol-Myers Squibb. Dr Sepesi has nothing to disclose with regard to commercial support.

Received for publication Sept 7, 2019; revisions received Sept 7, 2019; accepted for publication Sept 10, 2019; available ahead of print Oct 21, 2019.

Address for reprints: Boris Sepesi, MD, Department of Thoracic and Cardiovascular Surgery, The University of Texas MD Anderson Cancer Center, 1515 Holcombe Blvd, Houston, TX 77030 (E-mail: bsepesi@ mdanderson. org).

J Thorac Cardiovasc Surg 2020;159:1624-5

$0022-5223 / \$ 36.00$

Copyright (c) 2019 by The American Association for Thoracic Surgery

https://doi.org/10.1016/j.jtcvs.2019.09.042
}

The introduction of immune checkpoint inhibitors (ICIs) into the oncologic treatment armamentarium has created an unprecedented revolution in oncology not seen in decades. The addition of ICIs, including the antibodies directed against cytotoxic T-lymphocyte-associated protein 4 (CTLA-4), programmed cell death protein 1 (PD-1), and programmed death-ligand (PD-L1), to the existing anticancer regimens of chemotherapy, radiotherapy, and surgery creates an opportunity for a myriad of combinations to be studied in clinical trials. In metastatic non-small cell lung cancer (NSCLC), response rates, durability of responses, toxicity profile, and survival outcomes have demonstrated to be superior with ICIs alone, or in combination with chemotherapy in selected patients with expression of PD-L1 biomarker on tumor cells, relative to chemotherapy alone. ${ }^{1-3}$ In advanced surgically unresectable locoregional NSCLC, the addition of the anti-PD-L1 inhibitor durvalumab in the maintenance setting in patients who had a response or stability of the disease after definitive chemoradiation demonstrated superior survival and metastasis control relative to the control group. ${ }^{4,5}$

The review article in this issue of the Journal by Rhodin and colleagues, "The Immunotherapeutic Landscape in Non-Small Cell Lung Cancer and its Surgical Horizons," elegantly summarizes the current state of immunotherapy in NSCLC and speculates on the future of immunotherapy for surgical patients. The field of immunotherapy is rapidly evolving; however, granular and adequate patient-volume data, especially regarding the use of neoadjuvant ICIs and surgical outcomes in patients with NSCLC treated with these agents, have not yet made it into the published literature, although preliminary and in some cases final results from ongoing trials have been presented at major oncology

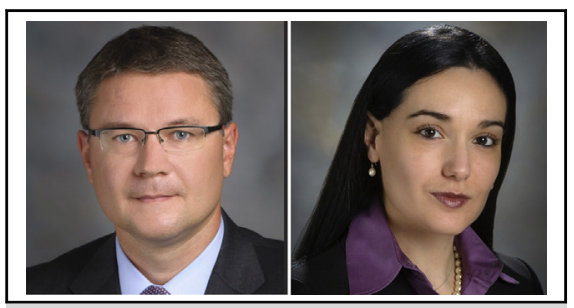

Boris Sepesi, MD (left), and Tina Cascone, MD, PhD (right)

\begin{abstract}
Central Message
Results from completed and ongoing neoadjuvant immunotherapy trials in lung cancer suggest that immunotherapy in preoperative setting is safe, feasible, and possibly more effective than chemotherapy alone, as determined by pathologic response rates.
\end{abstract}

See Article page 1616. meetings. To date, only a single feasibility neoadjuvant trial of 20 patients by Forde and associates ${ }^{7}$ has been published in its complete form. The trial demonstrated an unprecedented $45 \%$ major pathologic response (MPR) rate after only 2 cycles of nivolumab before surgery. Surgical outcomes of this trial were published by Bott and colleagues, ${ }^{8}$ who highlighted that there were no delays in resections, morbidity outcomes were comparable to those of neoadjuvant chemotherapy regimens, and, perhaps most importantly, a 54\% (7/13) conversion rate was seen from either a video-assisted thoracoscopic surgery or a robotic-assisted thoracoscopic surgery initial approach to thoracotomy in response to an "inflamed hilum." Communications among thoracic surgeons about anecdotal cases of inflammation after immunotherapy suggest that operating on patients in this setting is perhaps technically more challenging than operating primarily on early lung cancer. Importantly, though, perioperative outcomes are not negatively affected by this slightly increased technical challenge. ${ }^{8}$ The inflammatory reaction to ICIs appears to be idiosyncratic, and we currently do not have biomarkers that can predict intraoperative inflammation before surgical inspection of the pleural space.

In addition to the previously cited studies, 2 other recent neoadjuvant trials deserve highlighting. The first phase II randomized study to test nivolumab (anti-PD-1) as 
monotherapy or combined with ipilimumab (NEOSTAR, NCT03158129) has been completed. Results, which were presented by Cascone and coworkers ${ }^{9}$ at the American Society of Clinical Oncology annual meeting in 2019, demonstrated MPR rate of $17 \%$ (4/23) after 3 cycles of nivolumab and $33 \%(7 / 21)$ after combined nivolumab and ipilimumab in the intention-to-treat analysis. A novel phenomenon of nodal immune flare (NIF) has been observed in the NEOSTAR study, ${ }^{9}$ and detailed surgical outcomes from this trial ${ }^{10}$ and features of nodal immune flare ${ }^{11}$ were presented at the 2019 World Conference on Lung Cancer in Barcelona. Reports from NEOSTAR are forthcoming. The combination of ICIs and chemotherapy has been tested in ongoing and recently completed trials.

The NADIM trial (NCT03838159) $)^{12}$ has been completed, and its unprecedented results of combined $83 \%(34 / 41)$ MPR plus pathologic complete response in $71 \%(24 / 41)$ of patients with clinical stage IIIA disease were presented at the American Society of Clinical Oncology Annual Meeting in 2019. Reports from this trial are eagerly anticipated. The remainder of ongoing neoadjuvant trials have presented only interim analyses at this point, and the ANVIL (NCT02595944) adjuvant immunotherapy arm of the ALCHEMIST trial (NCT02194738) will take a few years to read out.

Checkpoint immunotherapy represents a significant advance in lung cancer oncology, and it is likely that ICIs will be eventually added to the neoadjuvant armamentarium of NSCLC. Many questions, however, still need to be answered. Will the ICIs be used according to biomarkers or for all-comers? How many induction cycles are most appropriate, and when is the best time for surgery to occur after induction immunotherapy? Should ICIs be continued in the adjuvant setting for all patients or for responders only, as determined by MPR, and for how long? Are neoadjuvant ICIs more efficacious given as monotherapy or dual therapy or in combination with chemotherapy or radiation? Does the tumor pathologic response rate translate into disease-free and overall survivals? Will MRP in the primary tumor become the Food and Drug Administration-approved surrogate of long-term survival in neoadjuvant trials? Does the response within the tumor and lymph nodes differ after neoadjuvant ICI-based strategies, and does nodal disease still represent the same metastatic risk during immunotherapy? There are certainly many more immunologic, clinical, and translational questions to be answered if we are to fine-tune the role of ICI therapy in resectable NSCLC. As surgeons, along with medical oncologists, we need to participate actively in this process and continue to support clinical trials that test immune-based therapies in the perioperative setting to answer these questions, and to refine the therapeutic options for patients with resectable lung cancer.

\section{References}

1. Brahmer JR, Govindan R, Anders RA, Antonia SJ, Sagorsky S, Davies MJ, et al. The Society for Immunotherapy of Cancer consensus statement on immunotherapy for the treatment of non-small cell lung cancer (NSCLC). J Immunother Cancer. 2018;6:75.

2. Mok TSK, Wu YL, Kudaba I, Kowalski DM, Cho BC, Turna HZ, et al; KEYNOTE-042 Investigators. Pembrolizumab versus chemotherapy for previously untreated, PD-L1-expressing, locally advanced or metastatic non-small-cell lung cancer (KEYNOTE-042): a randomised, open-label, controlled, phase 3 trial. Lancet. 2019;393:1819-30.

3. Reck M, Rodríguez-Abreu D, Robinson AG, Hui R, Csőszi T, Fülöp A, et al; KEYNOTE-024 Investigators. Pembrolizumab versus chemotherapy for PD-L1-positive non-small-cell lung cancer. N Engl J Med. 2016;375: 1823-33.

4. Antonia SJ, Villegas A, Daniel D, Vicente D, Murakami S, Hui R, et al; PACIFIC Investigators. Overall survival with durvalumab after chemoradiotherapy in stage III NSCLC. N Engl J Med. 2018;379:2342-50.

5. Antonia SJ, Villegas A, Daniel D, Vicente D, Murakami S, Hui R, et al; PACIFIC Investigators. Durvalumab after chemoradiotherapy in stage III non-small-cell lung cancer. N Engl J Med. 2017;377:1919-29.

6. Rhodin KE, Rucker JA, Ready NE, D'Amico TA, Antonia SJ. The immunother apeutic landscape in non-small cell cancer and its surgical horizons. J Thorac Cardiovasc Surg. 2020;159:1616-23.

7. Forde PM, Chaft JE, Smith KN, Anagnostou V, Cottrell TR, Hellmann MD, et al. Neoadjuvant PD-1 blockade in resectable lung cancer. N Engl J Med. 2018;378: 1976-86.

8. Bott MJ, Yang SC, Park BJ, Adusumilli PS, Rusch VW, Isbell JM, et al. Initial results of pulmonary resection after neoadjuvant nivolumab in patients with resectable non-small cell lung cancer. J Thorac Cardiovasc Surg. 2019;158: 269-76.

9. Cascone T, William W, Weissferdt A, Lin HY, Leung HC, Carter BW, et al. NEOSTAR phase II study of induction checkpoint blockade for untreated stage I-IIIA NSCLC amenable for surgical resection. J Clin Oncol. 2019;37(Suppl). Abstract 8504

10. Sepesi B, Cascone $\mathrm{T}$, William W, Lin $\mathrm{H}$, Leung $\mathrm{CH}$, Weissferdt $\mathrm{A}$, et al. Surgical outcomes following neoadjuvant nivolumab or nivolumab plus ipilimumab in non-small cell lung cancer-NEOSTAR Study. Abstract OA13.06. Presented at: World Conference on Lung Cancer; September 7-10, 2019; Barcelona, Spain.

11. Sepesi B, Godoy MC, William WN, Vaporciyan AA, Lin H, Lee J, et al. Nodal immune flare (NIF) following anti-PD-1 and anti-CTLA-4 therapy in non-small cell lung cancer. Abstract P2.04-90. Presented at: World Conference on Lung Cancer; September 7-10, 2019; Barcelona, Spain.

12. Provencio M, Nadal E, Insa A, Garcia-Campelo R, Rubio JC, Domine M, et al. NEO-adjuvant chemo-immunotherapy for the treatment of STAGE IIIA resectable non-small-cell lung cancer (NSCLC): a phase II multicenter exploratory study - final data of patients who underwent surgical assessment. J Clin Oncol. 2019;37(Suppl). Abstract 8509. 